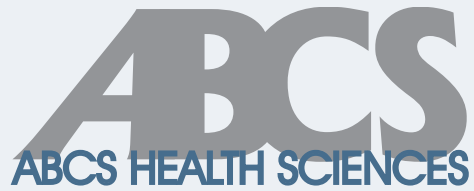

How to cite this article: Nishi et al. Postoperative complications of penetrating corneal keratoplasty in patients with anterior segment toxic syndrome secondary to cataract surgery. ABCS Health Sci. 2020;45(Suppl.3):e020105. https://doi. org/10.7322/abcshs.2020S05.1852

Corresponding Author: Vagner Loduca Lima - Discipline of Ophthalmology, Centro Universitário FMABC - Avenida Lauro Gomes, 2000 - Sacadura Cabral - CEP 09060-650 - Santo André (SP), Brazil -

E-mail:vagner@loduca.com.br

Declaration of interests: nothing to declare

This is an open access article distributed under the terms of the Creative Commons Attribution License

(C) 2020 Nishi et al.

\title{
Post-operative complications
} of penetrating corneal keratoplasty in patients with anterior segment toxic syndrome secondary to cataract surgery

Elen Nishimoto Nishi ${ }^{1}$, Vagner Loduca Lima ${ }^{1}$, Glaucia Luciano da Veiga ${ }^{1,2}$, Luiz Antonio de Brito Martins ${ }^{1}$

${ }^{1}$ Discipline of Ophthalmology. Centro Universitário FMABC - Santo André (SP), Brazil ${ }^{2}$ Clinical Analysis Laboratory, Centro Universitário FMABC - Santo André (SP), Brazil

\begin{abstract}
Introduction: Toxic Anterior Segment Syndrome (TASS) after cataract surgery may cause severe corneal decompensation that compromises corneal transparency and may require penetrating corneal keratoplasty to improve visual acuity. Objective: We evaluated the postoperative complications of patients who underwent penetrating corneal transplantation for severe corneal decompensation secondary to TASS after cataract surgery, such as persistent epithelial defect, glaucoma, and primary and secondary transplant button failure. We will also evaluate pre- and postoperative visual acuity, graft survival time, and the presence of anterior chamber disorganization. Methods: Retrospective observational study in which a review of medical records of 9 patients diagnosed with TASS after cataract surgery who underwent penetrating corneal keratoplasty will occur. Results: In the present study all operated patients had glaucoma after penetrating corneal transplantation, and this presence of glaucoma was not correlated with graft survival time and with any other parameter evaluated. The presence of persistent epithelial defect correlated negatively with visual acuity. Conclusion: Postoperative complications of penetrating corneal transplantation in patients with TASS were frequent, such as glaucoma, primary and secondary button failure and persistent epithelial defect. The only complication that compromised visual acuity was the persistent epithelial defect. All patients evolved with glaucoma.
\end{abstract}

Keywords: corneal transplantation; anterior segment toxic syndrome.

\section{INTRODUCTION}

Toxic anterior segment syndrome (TASS) is a complication caused by an acute, noninfectious inflammatory reaction to substances used in ophthalmic surgery. It causes both intracellular and extracellular damage to intraocular tissues. This syndrome is characterized by visual blurring, limbus-to-limb corneal edema, involvement of the anterior segment only, fibrin formation, hypopyon, irregular or fixed pupil. The onset of the condition occurs early postoperatively, usually 24 to 48 hours after surgery. But there are reports in the literature of late presentation, with onset up to 137 days postoperatively ${ }^{1}$. 
Its diagnosis is clinical. It must have negative culture for fungi and bacteria and negative polymerase chain reaction (PCR) 2 .

It responds well to topical steroid treatment. Subconjunctival injection of steroids or intracameral injection of a thrombolytic agent such as recombinant human tissue plasminogen activator ( $\mathrm{rtPA}$ ) can be performed. The exact incidence of TASS and its risk factors are unknown. There are reports of a variable incidence of 0.1 to $2.0 \%{ }^{3}$.

Some systemic diseases are more common in patients with TASS, such as type 2 diabetes mellitus, hypertension, dyslipidemia, heart disease, and chronic renal failure ${ }^{4}$.

TASS causes corneal decompensation by the inflammatory condition that breaks down the tight junctions between endothelial cells with consequent corneal edema. Severe corneal decompensation may require corneal transplantation. Another complication of TASS is increased intraocular pressure (IOP) due to fibrin membrane formation and trabeculitis that compromises trabecular filtration. Chronic lesions can cause secondary glaucoma.

Patients were referred to our service for evaluation and management of corneal decompensation secondary to TASS after cataract surgery. Due to the severity of the condition, these patients had indication for penetrating corneal keratoplasty. The surgeries were performed under general anesthesia and without intercurrences at Mário Covas State Hospital in Santo André in the period from $12 / 07 / 2017$ to $07 / 30 / 2019$. And during postoperative follow-up it was observed that the patients evolved with glaucoma of difficult clinical control, persistent epithelial defect and primary and secondary failure of the transplant button.

The objective of this study was to report the postoperative complications of patients who underwent a penetrating keratoplasty in the eye affected by TASS after cataract surgery. We also evaluated the presence of anterior chamber disorganization and whether there is a correlation with transplant survival time.

\section{METHODS}

A retrospective observational study that reviewed medical records of 9 patients diagnosed with TASS after cataract surgery who had severe corneal decompensation and underwent penetrating corneal keratoplasty in the Centro Universitário FMABC. We evaluated epidemiological data such as patient age at the time of surgery, gender, and comorbidities. We compared the pre- and postoperative ophthalmologic picture, such as visual acuity (VA), glaucoma, persistent epithelial defect, and survival time of the transplant button.

The data were expressed as percentage and mean \pm standard deviation of the mean (SD) and were analyzed in GraphPad Prism software version 6.0. To evaluate the post-surgery effects the repeated measures ANOVA test was performed and the correlation evaluation was done using Spearman's test. The values considered statistically significant were those with $\mathrm{p}$-value $<0.05$.

\section{RESULTS}

Nine eyes of patients undergoing corneal transplantation for corneal decompensation after TASS of Centro Universitário FMABC from 12/07/2017 to 7/30/2019 were analyzed.

In our study there was a higher incidence of TASS in males (Table 1). Hypertension was the most prevalent systemic disease (4 patients), followed by diabetes mellitus and hypothyroidism ( 2 patients).

The average age of the patients was 73.4 years, ranging from 65 to 83 years.

Two patients (22\%) had a persistent epithelial defect.

In 3 patients we found primary transplant failure (33\%) and only 1 had secondary failure (11\%).

Over $70 \%$ had anterior chamber disorganization, including iris atrophy, anterior synechiae, and paralytic mydriasis.

In the present study all operated patients had glaucoma after corneal penetrant transplantation, and this presence of glaucoma was not correlated with graft survival time and with any other parameter evaluated.

The presence of persistent epithelial defect had a negative correlation with visual acuity $(\mathrm{p}<0.0001 \mathrm{r}=-0.3258, \mathrm{n}=9)$.

In the evaluation of visual acuity, there was no difference between the preoperative visual acuity and the visual acuity at 1 and 6 months postoperatively. However, in the 3 -month postoperative period, visual acuity worsened with statistical significance (Figure 1).

Two patients were left with visual acuity without light perception. One of them due to glaucoma and the other due to retinal detachment. One of the patients presented hyphemia in the anterior chamber (Figure 2). Four patients had to undergo filtering surgery for glaucoma control.

Table 1: Analysis of the results

\begin{tabular}{|l|c|c|}
\hline Parameters & Male (55.5\%) & Female (44.4\%) \\
\hline Gender & $73.4 \pm 6.6$ \\
\hline Age (years) & $22 \%$ \\
\hline Persistent epithelial defect (\%) & $55 \%$ \\
\hline Preoperative Glaucoma (\%) & $100 \%$ \\
\hline Postoperative Glaucoma (\%) & $33 \%$ \\
\hline Primary failure (\%) & $11 \%$ \\
\hline Secondary failure (\%) & $77 \%$ \\
\hline Anterior chamber depth (\%) & $221 \pm 126$ \\
\hline Survival time (days) & $8 \pm 1$ \\
\hline Preoperative VA & $6 \pm 2$ \\
\hline VA after 1 month of surgery & $5 \pm 3$ \\
\hline VA after 3 months of surgery & $7 \pm 3$ \\
\hline VA after 6 months of surgery & \\
\hline
\end{tabular}

VA: visual acuity 


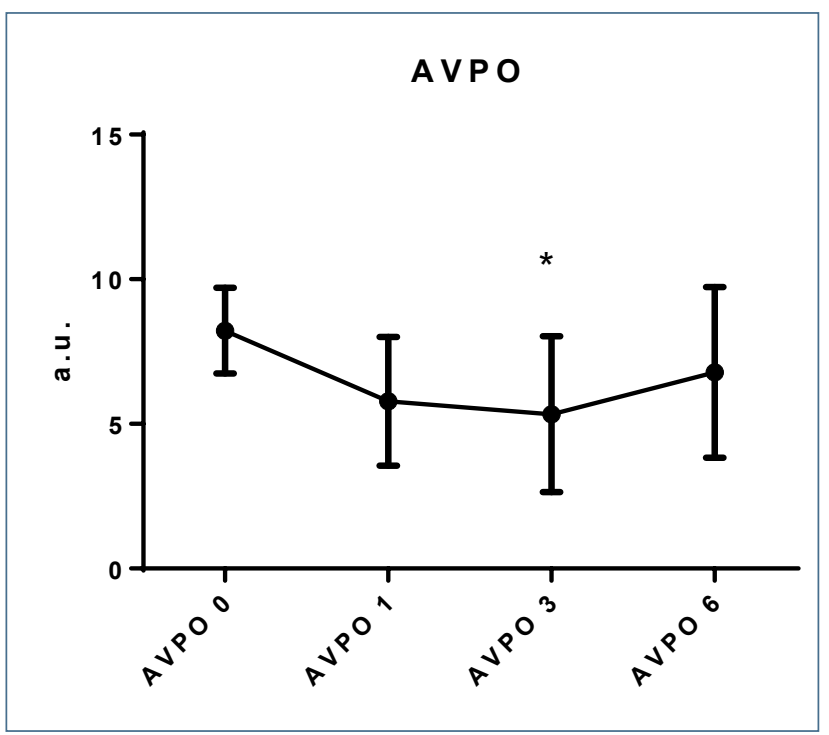

Figure 1: Comparison of preoperative (AVPO) and postoperative visual acuity at 1 (AVP1), 3 (AVP2) and 6 (AVPO6) months. Repeated measures ANOVA test. * $p<0.05$ AVPO vs AVPO0.

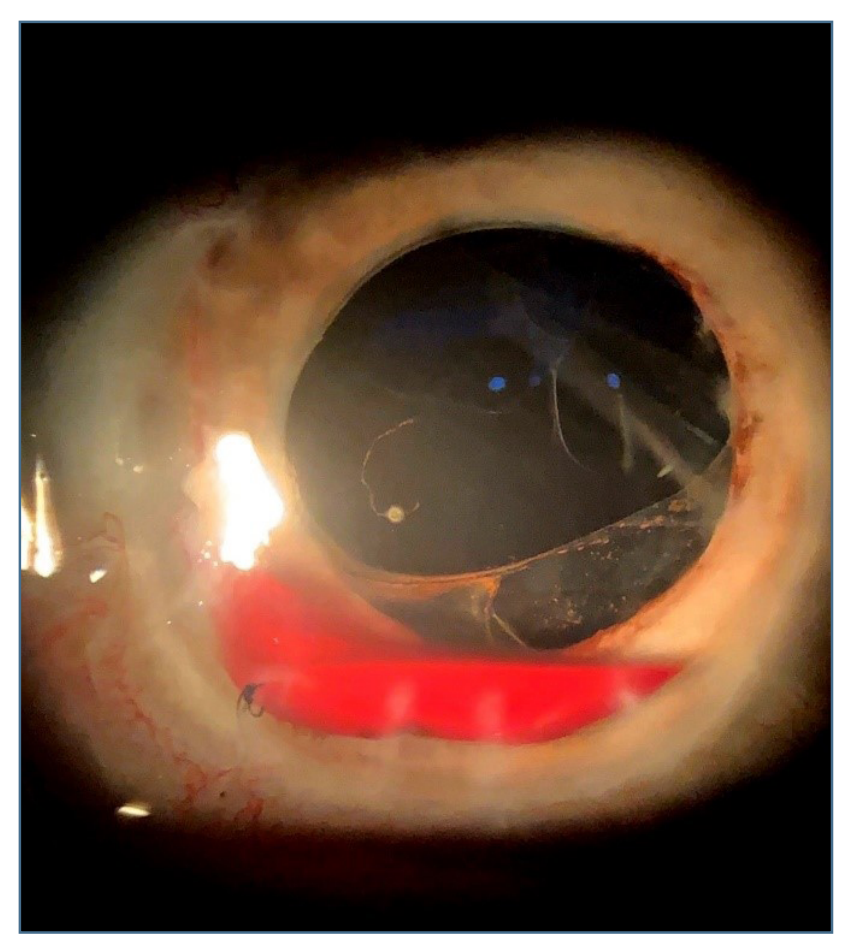

Figure 2: Hyphemia in anterior chamber.

\section{DISCUSSION}

In the present study we observed a high incidence of postoperative complications in patients undergoing penetrating corneal keratoplasty after corneal decompensation by TASS. We found no reports in the literature of these complications.

Corneal decompensation occurs due to endothelial damage and lamellar transplants such as Descemet's automated denuding endothelial keratoplasty (DSAEK) is reported to be the procedure of choice for treating patients with TASS ${ }^{5}$. Our patients had structural compromise of all corneal layers that did not allow lamellar transplants to be performed, so our procedure of choice was penetrating keratoplasty.

All our patients evolved with glaucoma in the postoperative follow-up. Four of them did not respond adequately to clinical treatment and had to undergo filtering surgery for glaucoma control. One patient lost his vision due to glaucoma and another patient lost his vision due to total retinal detachment.

During the postoperative follow-up, the patients showed a reduction in visual acuity in the third month. But it was recovered by the sixth postoperative month.

Glaucoma, primary and secondary transplant button failures, and anterior chamber disorganization did not correlate with statistical significance with visual acuity ${ }^{5-9}$. The only postoperative complication that showed a negative correlation with visual acuity was the presence of a persistent epithelial defect.

\section{Conclusion}

Patients presenting with corneal decompensation due to TASS had frequent postoperative complications of penetrating corneal transplantation, such as glaucoma, primary and secondary button failure, and persistent epithelial defect. The only complication that compromised visual acuity was the persistent epithelial defect. All patients evolved with glaucoma, and two lost vision.

There was a reduction in visual acuity with statistical significance in the third postoperative month. But by the sixth month the patients had recovered this difference.

Our work is pioneer in discussing these complications. There is still a need for follow-up, because visual acuity has a good prognosis of improvement in these patients.

\section{REFERENCES}

1. Miyake G, Ota I, Miyake K, Zako M, Iwaki M, Shibuya A. Lateonset toxic anterior segment syndrome. J Cataract Refract Surg. 2015;41(3):666-9.

https://doi.org/10.1016/j.jcrs.2015.01.002

2. Holland SP, Morck DW, Lee TL. Update on toxic anterior segment syndrome. Curr Opin Ophthalmol. 2007;18(1):4-8 https://doi.org/10.1097/ICU.0b013e3280117d0c
3. Sengupta S, Chang DF, Gandhi R, Kenia H, Venkatesh R. Incidence and long-term outcomes of toxic anterior segment syndrome at Aravind Eye Hospital. J Cataract Refract Surg. 2011;37(9):1673-8. https://doi.org/10.1016/j.jcrs.2011.03.053

4. Yazgan S, Celik U, Ayar O, Ugurbas SH, Celik B, Akdemir $\mathrm{MO}$, et al. The role of patient's systemic characteristics and plateletcrit in developing toxic anterior segment syndrome after 
uneventful phaco surgery: A case-control study. Int Ophthalmol. 2018;38(1):43-52.

https://doi.org/10.1007/s10792-016-0418-x

5. Arslan OS, Unal M, Arici C, Görgün E, Yenerel M, Cicik E. Descemet-stripping automated endothelial keratoplasty in eyes with toxic anterior segment syndrome after cataract surgery. J Cataract Refract Surg. 2010;36(6):965-9.

https://doi.org/10.1016/j.jcrs.2009.12.037

6. Sevimli N, Karadag R, Cakici O, Bayramlar H, Okumus S, Sari U. Toxic anterior segment syndrome following deep anterior lamellar keratoplasty. Arq Bras Oftalmol. 2016;79(5):330-2. https://doi.org/10.5935/0004-2749.20160094
7. Shouchane-Blum K, Dotan A, Bahar I. The evolution of toxic anterior segment syndrome. Curr Opin Ophthalmol. 2019;30(1):50-5.

https://doi.org/10.1097/ICU.0000000000000540

8. Bodnar Z, Clouser S, Mamalis N. Toxic anterior segment syndrome: Update on the most common causes. J Cataract Refract Surg. 2012;38(11):1902-10.

https://doi.org/10.1016/j.jcrs.2012.06.053

9. Park CY, Lee JK, Chuck RS. Toxic anterior segment syndrome-an updated review. BMC Ophthalmol. 2018;18(1):276.

https://doi.org/10.1186/s12886-018-0939-3 